УДК 621.382

\title{
УСИЛЕНИЕ ОПТИЧЕСКИХ ФОНОНОВ В УЗКОЗОННЫХ ПОЛУПРОВОДНИКАХ ПРИ НИЗКИХ ТЕМПЕРАТУРАХ
}

\author{
КАСТРЕХОН-М. К., ГРИМАЛЬСКИЙ В. В., КОШЕВАЯ С. В., ТЕКПОЙОТЛЬ-Т. М.
}

Автономный университет штата Морелос, Мексика, Куэрнавака, Z. Р. 62209

\begin{abstract}
Аннотация. Исследована генерация терагерцового излучения при использовании собственных мод твердого тела. Произведено численное моделирование неустойчивости оптических фононов терагерцового диапазона в полупроводниковых структурах с квантовыми ямами при дрейфе двумерного электронного газа. Основным препятствием для реализации данной неустойчивости является нагрев электронного газа в процессе дрейфа. Поэтому рассмотрены низкие температуры $T<77$ К, и электронный газ предполагается вырожденным. Благодаря существующим микроохладителям замкнутого цикла, а также реализации обратной связи имеется возможность экспериментально наблюдать генерацию оптических фононов. При численном моделировании использовались как кинетический, так и гидродинамический подходы. Показано, что кинетический подход более адекватен, тогда как гидродинамический дает завышенные значения инкрементов неустойчивости
\end{abstract}

Ключевые слова: терагерцовое излучение; двумерный электронный газ; дрейф; усиление

\section{1. ВВЕДЕНИЕ}

Излучение промежуточного диапазона между миллиметровым и оптическим диапазонами, так называемый терагерцовый диапазон $f=$ 0,1-30 ТГц, сейчас используется в различных областях науки, техники и медицины $[1,2]$.

В настоящее время практически исчерпаны все возможности получить терагерцовое излучение без применения сложных вакуумных устройств, таких как лампа обратной волны, гиротрон и т.д. Лазерные методы столкнулись с целым рядом сложных конструктивных и физических проблем. Более того, их сопряжение с широко применяемыми в миллиметровом и субмиллиметровом диапазонах твердо- тельными устройствами, такими как диоды и транзисторы, практически недоступны.

Излучение терагерцового диапазона имеет огромные перспективы практического использования. Например, в сканерах аэропортов, где до сих пор используется опасное для здоровья рентгеновское излучение. Таким образом, поиск неустойчивостей, которые могут быть полезны для создания терагерцовых твердотельных устройств, возможен при использовании собственных колебаний и волн кристаллической решетки, таких как оптические фононы (полярные колебания решетки) в полупроводниках [3, 4]. Механизм неустойчивости оптических фононов является черен-

* Работа частично поддержана SEP-CONACyT (Mexico). 
ковским, при котором фазовая скорость усиливаемой волны меньше скорости носителей [5]. При этом важно коллективное взаимодействие электронов с оптическими фононами.

В данной работе теоретически исследована неустойчивость оптических фононов в полупроводниковых структурах с квантовыми ямами в присутствии дрейфа электронного газа, которая может привести к генерации колебаний терагерцового диапазона при соответствующей конструкции. Двумерный электронный газ локализован в одном направлении.

Препятствием для наблюдения генерации оптических фононов является нагрев электронного газа при дрейфе, что ведет к увеличению давления газа и к росту частоты столкновения. Поэтому рассмотрены низкие температуры $T<77$ К и электронный газ рассмотрен как вырожденный. Учет давления электронного газа является принципиальным.

Поскольку анализируется терагерцовый диапазон, проведен анализ неустойчивости двумя методами, квантовым кинетическим и гидродинамическим. Следует отметить, что в данной работе принципиальное значение имеет анализ наличия самой неустойчивости с приемлемыми для практики значениями инкрементов нарастания во времени. Нелинейная стадия нарастания сигнала требует совершенно другого подхода и не является целью данной работы.

Отметим также, что объемные полупроводниковые структуры практически неприменимы для реализации неустойчивости из-за невозможности понизить частоты столкновения электронного газа, и, следовательно, быстрого разогрева при дрейфе.

Конструкция генератора основана на использовании планарной структуры с диэлектрическими входом и выходом аналогично $[6,7]$.

\section{2. ОСНОВНЫЕ УРАВНЕНИЯ}

В квантовом кинетическом методе уравнение движения двумерного электронного газа в квантовой яме есть уравнение одночастичной мат- рицы плотности $\hat{\rho}$. Ось $O X$ направлена перпендикулярно яме, оси $O Y, O Z$ - вдоль нее. Дрейф электронного газа происходит вдоль оси $O Z$.

В координатном представлении это уравнение имеет вид [8-13]:

$$
\begin{gathered}
\mathrm{i} \hbar \frac{\partial \rho\left(\vec{r}_{1}, \vec{r}_{2}, t\right)}{\partial t}=-\frac{\hbar^{2}}{2 m^{*}}\left(\Delta_{r_{1}}-\Delta_{r_{2}}\right) \rho\left(\vec{r}_{1}, \vec{r}_{2}, t\right)+ \\
+e\left(W_{0}\left(x_{1}\right)+\hat{V}_{0}\left(z_{1}\right)+\hat{\widetilde{V}}\left(\vec{r}_{1}, t\right)-W_{0}\left(x_{2}\right)-\right. \\
\left.-\hat{V}_{0}\left(z_{2}\right)-\hat{\tilde{V}}\left(\vec{r}_{2}, t\right)\right) \rho\left(\vec{r}_{1}, \vec{r}_{2}, t\right)- \\
-i \hbar v_{\mathrm{p}}\left(\rho\left(\vec{r}_{1}, \vec{r}_{2}, t\right)-\rho_{0}\left(\vec{r}_{1}, \vec{r}_{2}\right)\right)
\end{gathered}
$$

где $\hat{V}_{0}=-e E_{0} z, \hat{\widetilde{V}}=e \widetilde{\varphi}$ — части гамильтониана, обусловленные приложенным постоянным и самосогласованным переменным электрическими полями; $\hat{\rho}_{0}$ - стационарная матрица плотности, вид которой обсуждается ниже; $W_{0}(x)$ - потенциальная энергия электрона в квантовой яме; $m^{*}$ — эффективная масса электрона, i — мнимая единица. Релаксационный член учитывается в приближении частоты релаксации $v_{\mathrm{p}}$.

В случае линейных волн используется выражение

$$
\rho\left(\vec{r}_{1}, \vec{r}_{2}, t\right)=\rho_{0}\left(\vec{r}_{1}, \vec{r}_{2}\right)+\widetilde{\rho}\left(\vec{r}_{1}, \vec{r}_{2}, t\right),
$$

где $\widetilde{\rho}$ - малое переменное возмущение матрицы плотности.

Используя одночастичную матрицу плотности, возможно рассчитать электронную концентрацию

$$
n\left(\vec{r}_{1}\right)=2 \rho\left(\vec{r}_{1}, \vec{r}_{1}\right) \equiv \operatorname{Sp}\left\{\hat{\rho} \hat{n}^{(1)}\left(\vec{r}_{1}\right)\right\}
$$

где $\hat{n}^{(1)}\left(\vec{r}_{1}\right)=\delta\left(\vec{r}-\vec{r}_{1}\right)$ - одночастичный оператор электронной концентрации; множитель «2» в (2) учитывает спин электрона; Sp - след оператора, т.е. сумма его диагональных матричных элементов $[9,12]$. 
Вдоль оси $O X$ движение электрона квантовано, уровни энергии описываются индексами $\alpha, \beta$. Вдоль осей $O Y, O Z$ движение квазиклассическое: $\vec{r}_{\|} \equiv(y, z)$.

Далее используем матрицу плотности в смешанном координатно-импульсном представлении, так называемая функция распределения Вигнера (ФВ) $f_{\alpha \beta}\left(\vec{r}_{\|}, \vec{p}, t\right)$ [9-12], которая получается из матрицы плотности $\rho\left(\vec{r}_{1}, \vec{r}_{2}, t\right)$ при замене переменных $\vec{r}_{\|}=\left(\vec{r}_{1 \|}+\vec{r}_{2 \|}\right) / 2, \vec{R}_{\|}=\vec{r}_{1 \|}$ $-\vec{r}_{2 \|}$ и дальнейшем преобразовании Фурье по переменной $\vec{R}_{\|}$, а также разложении по волновым функциям в квантовой яме $\psi_{\alpha}(x)$.

Ортонормированные волновые функции для электрона в квантовой яме могут быть выбраны действительными:

$$
\int_{-\infty}^{+\infty} \psi_{\alpha}(x) \psi_{\beta}(x) \mathrm{d} x=\delta_{\alpha \beta}
$$

Функция Вигнера - аналог одночастичной функции распределения в классической кинетике $[11,12]$. Для линейных волн ФВ возможно представить в виде

$$
f_{\alpha \beta}=f_{0 \alpha}(\vec{p}) \delta_{\alpha \beta}+\tilde{f}_{\alpha \beta}(z, \vec{p}, t),
$$

где $f_{0 \alpha}-$ стационарная функция распределения, $\tilde{f}-$ переменное возмущение; $\vec{p} \equiv\left(p_{y}, p_{z}\right)$ - квазиимпульс электрона.

Переменные части $\widetilde{n}, \widetilde{\varphi}, \widetilde{f}$ ищутся в виде $\widetilde{n}, \widetilde{\varphi}, \widetilde{f} \sim \exp (-\mathrm{i} \omega t+\mathrm{i} k z)$. При поиске неустойчивости волновое число $k$ действительно, тогда как частота комплексная [5] $\omega=\omega^{\prime}+\mathrm{i} \omega^{\prime \prime}$. В случае неустойчивости мнимая часть частоты положительна $\omega^{\prime \prime}>0$.

Используем приближение, в котором стационарная $Ф \mathrm{~B}$, или стационарная функция распределения электронов, аппроксимируется смещенным распределением Ферми:

$$
f_{0 \alpha}(\vec{p})=\theta\left(E_{\Phi}-E_{\alpha}(\vec{p})\right)
$$

$$
\begin{gathered}
E_{\alpha}(\vec{p})=E_{\alpha 0}+\hbar^{2} \frac{p_{y}^{2}+\left(p_{z}-p_{0}\right)^{2}}{2 m^{*}}, \\
p_{0}=\frac{m^{*} v_{0}}{\hbar}
\end{gathered}
$$

где $\theta(E)$ - ступенчатая функция, $E_{\Phi}-$ энергия Ферми.

В (4) предполагается, что приложенное постоянное электрическое поле $E_{0}<500 \mathrm{~B} /$ см не настолько высоко, чтобы модифицировать электронные уровни энергии в зоне проводимости. Влияние поля смещения $E_{0}$ проявляется только при формировании смещенной стационарной функции распределения, которая соответствует скорости дрейфа $v_{0}$.

В данном приближении уравнение для переменной части матрицы плотности представляется как [12]:

$$
\begin{gathered}
{\left[\hbar\left(\omega+\mathrm{i} v_{\mathrm{p}}\right)-E_{\alpha}\left(\vec{p}+\frac{\vec{k}}{2}\right)+E_{\beta}\left(\vec{p}-\frac{\vec{k}}{2}\right)\right] \widetilde{f}_{\alpha \beta}(\vec{p})=} \\
=-e \widetilde{\varphi}_{\alpha \beta}\left[f_{0 \beta}\left(\vec{p}-\frac{\vec{k}}{2}\right)-f_{0 \alpha}\left(\vec{p}+\frac{\vec{k}}{2}\right)\right] \\
\widetilde{\varphi}_{\alpha \beta}=\int_{-\infty}^{+\infty} \widetilde{\varphi}(x) \psi_{\alpha}(x) \psi_{\beta}(x) \mathrm{d} x
\end{gathered}
$$

Для переменного электрического потенциала $\widetilde{\varphi}$ используется уравнение Пуассона

$$
\nabla \cdot(\varepsilon(\omega) \vec{\nabla} \widetilde{\varphi})=-\frac{e}{\varepsilon_{0}} \widetilde{n} ; \quad \widetilde{\vec{E}}=-\vec{\nabla} \widetilde{\varphi},
$$

где $\varepsilon(\omega)$ - решеточная диэлектрическая проницаемость проводника; $\varepsilon_{0}$ - электрическая постоянная в системе СИ. Здесь переменная электронная концентрация $\widetilde{n}$ выражена через возмущение ФВ $\tilde{f}$ :

$$
\widetilde{n}(x)=\frac{1}{2 \pi^{2}} \sum_{\alpha, \beta} \psi_{\alpha}(x) \psi_{\beta}(x) \int \widetilde{f}_{\alpha \beta}(\vec{p}) \mathrm{d} p_{y} \mathrm{~d} p_{z} .
$$


Интегрирование по квазиимпульсу имеет место по всей плоскости.

Рассмотрим приближение, когда заселенность нижнего уровня в квантовой яме преобладает [14] $\alpha=\beta=1$ :

$$
f_{10}(\vec{p})=\theta\left(p_{\Phi}^{2}-\left(p_{z}-p_{0}\right)^{2}-p_{y}^{2}\right) .
$$

Импульс Ферми может быть выражен через концентрацию двумерного электронного газа $p_{\Phi}=\left(2 \pi n_{02}\right)^{1 / 2}$.

Для переменной части ФВ $\widetilde{f}$ из (5) получим следующее выражение $[10,11]$ :

$$
\begin{gathered}
\tilde{f}_{11}(\vec{p})=-\frac{e \widetilde{\varphi}_{11}}{\hbar\left(\omega-k v_{z}+\mathrm{iv} v_{\mathrm{p}}\right)} \times \\
\times\left[f_{10}\left(\vec{p}+\frac{\vec{k}}{2}\right)-f_{10}\left(\vec{p}-\frac{\vec{k}}{2}\right)\right], \\
\widetilde{\varphi}_{11}=\int_{-\infty}^{+\infty} \widetilde{\varphi}(x) \psi_{1}^{2}(x) \mathrm{d} x .
\end{gathered}
$$

Отличие (9) от результатов $[12,13]$ состоит в использовании смещенной функции Ферми (8) вместо обычной. Здесь волновой вектор волны равен $\vec{k}=(0,0, k)$. Столкновительный член аппроксимирован как релаксационный $-v_{\mathrm{p}} \tilde{f}$ [10].

Записав уравнение Пуассона (6) как:

$$
\frac{\mathrm{d}^{2} \widetilde{\varphi}}{\mathrm{d} x^{2}}-k^{2} \widetilde{\varphi}=-\frac{e \widetilde{n}(x)}{\varepsilon_{0} \varepsilon(\omega)}
$$

и выполнив интегрирование [12] получаем выражение для электрического потенциала волны $\widetilde{\varphi}$ :

$$
\widetilde{\varphi}(x)=\frac{e}{2 \varepsilon_{0} \varepsilon(\omega) k} \int_{-\infty}^{+\infty} \exp \left(-k\left|x-x^{\prime}\right|\right) \widetilde{n}\left(x^{\prime}\right) \mathrm{d} x^{\prime},
$$

$$
\widetilde{n}(x)=\frac{1}{2 \pi^{2}} \psi_{1}^{2}(x) \int \tilde{f}_{11}(\vec{p}) \mathrm{d} p_{y} \mathrm{~d} p_{z}
$$

$$
M_{11}=\int_{-\infty}^{+\infty} \int_{-\infty}^{+\infty} \exp \left(-k\left|x-x^{\prime}\right|\right) \psi_{1}^{2}(x) \psi_{1}^{2}\left(x^{\prime}\right) \mathrm{d} x \mathrm{~d} x^{\prime} .
$$

Таким образом получаем:

$$
\begin{gathered}
\widetilde{\varphi}_{11}=-\frac{e}{2 \varepsilon_{0} \varepsilon(\omega) k} M_{11} \frac{1}{2 \pi^{2}} \frac{e \widetilde{\varphi}_{11}}{\hbar} \times \\
\times \int \frac{f_{10}\left(\vec{p}+\frac{\vec{k}}{2}\right)-f_{10}\left(\vec{p}-\frac{\vec{k}}{2}\right)}{\omega-k v_{z}+\mathrm{i} v} \mathrm{~d} p_{y} \mathrm{~d} p_{z},
\end{gathered}
$$

где $M_{11}$ - интеграл перекрытия между волновой функцией и переменным электрическим потенциалом.

Интеграл перекрытия оценивался для различных квантовых ям и принимался равным 0,5. Из (10), (11) получаем следующее дисперсионное уравнение:

$$
\begin{gathered}
\varepsilon(\omega)+\frac{e^{2} m^{*}}{2 \pi \varepsilon_{0} \hbar^{2} k^{2}} M_{11} \frac{1}{2 \pi^{2}} \times \\
\times \int \frac{f_{10}\left(\vec{p}+\frac{\vec{k}}{2}\right)-f_{10}\left(\vec{p}-\frac{\vec{k}}{2}\right)}{\frac{m^{*}\left(\omega+\mathrm{i} v_{\mathrm{p}}\right)}{k \hbar}-p_{z}} \mathrm{~d} p_{y} \mathrm{~d} p_{z}=0 .
\end{gathered}
$$

Выражение для $\varepsilon(\omega)$ в кристаллах кубической симметрии в пренебрежении пространственной дисперсией [15] имеет вид:

$$
\begin{gathered}
\varepsilon(\omega)=\varepsilon(\infty)+\frac{\varepsilon(0)-\varepsilon(\infty)}{1-\left(\omega / \omega_{\mathrm{T}}\right)^{2}-\mathrm{i} \gamma \omega / \omega_{\mathrm{T}}^{2}}, \\
\omega_{\mathrm{L}}=\omega_{\mathrm{T}}(\varepsilon(0) / \varepsilon(\infty))^{1 / 2}
\end{gathered}
$$

где $\omega_{\mathrm{T}}, \omega_{\mathrm{L}}$ - частоты колебаний поперечных (T) и продольных (L) оптических фононов, $\gamma-$ диссипация фононов, $\varepsilon(0), \varepsilon(\infty)$ - низко- и вы- 
сокочастотная решеточные проницаемости $[3,15]$.

Далее рассмотрены значения волновых чисел $k \leq 2 \times 10^{8} \mathrm{M}^{-1}$, которые намного меньше пределов зоны Бриллюэна $\pi / a \approx 6 \times 10^{9} \mathrm{M}^{-1}$, где $a \approx 0,5 \mathrm{Hм}-$ период кристаллической решетки. Поправки в (7) в знаменателе из-за пространственной дисперсии примерно равны $(k a / \pi)^{2}<10^{-3}$ [15].

Интеграл в (12) может быть вычислен аналитически:

$$
\begin{gathered}
\frac{1}{2 \pi^{2}} \int \frac{f_{10}\left(\vec{p}+\frac{\vec{k}}{2}\right)}{\frac{m^{*}\left(\omega+\mathrm{i} v_{\mathrm{p}}\right)}{k \hbar}-p_{z}} \mathrm{~d} p_{y} \mathrm{~d} p_{z}= \\
=\frac{p_{\Phi}}{\pi}\left[a_{1}+\left(1-a_{1}^{2}\right) I\left(a_{1}\right)\right] \\
a_{1,2}=\frac{I(a)=\frac{1}{2 \pi} \int_{0}^{2 \pi} \frac{\mathrm{d} \theta}{a-\cos \theta}}{p_{\Phi}} ; \quad p_{1,2}=\frac{m^{*}\left(\omega+\mathrm{i} \nu_{\mathrm{p}}\right)}{k \hbar}-p_{0} \pm \frac{k}{2} .
\end{gathered}
$$

После чего дисперсионное уравнение возможно записать как

$$
\begin{gathered}
\varepsilon(\omega)+\frac{e^{2}}{2 \pi \varepsilon_{0} m^{*}\left(\frac{\hbar k}{m^{*}}\right)^{2}} M_{11}\left\{a_{1}+\right. \\
\left.+\left(1-a_{1}^{2}\right) I\left(a_{1}\right)-a_{2}-\left(1-a_{2}^{2}\right) I\left(a_{2}\right)\right\}=0 .
\end{gathered}
$$

Данное дисперсионное уравнение описывает гибридную электрон-фононную волну в присутствии дрейфа электронов.

Для вычисления интеграла I( $a$ ) используется правило Ландау [12]. Этот интеграл вычисляется при помощи контурного интегрирования в комплексной плоскости, а полюса подынтегрального выражения выбраны из условия, что $\omega+\mathrm{i} v_{\mathrm{p}}$ имеет положительную мнимую часть.
Неустойчивость также рассмотрена при помощи более простого гидродинамического подхода. Система линеаризованных гидродинамических уравнений для возмущений двумерной электронной концентрации $\tilde{n}_{2}$ и скорости $\widetilde{\vec{v}}$ имеет вид [8]:

$$
\begin{gathered}
\frac{\partial \widetilde{n}_{2}}{\partial t}+\vec{v}_{0} \cdot \vec{\nabla} \widetilde{n}_{2}+n_{0} \vec{\nabla} \widetilde{\vec{v}}=0, \\
\frac{\partial \widetilde{\vec{v}}}{\partial t}+\left(\vec{v}_{0} \cdot \vec{\nabla}\right) \widetilde{\vec{v}}=\frac{e}{m^{*}} \widetilde{\vec{E}}-v_{s}^{2} \vec{\nabla} \widetilde{n}_{2}-v_{\mathrm{p}} \widetilde{\vec{v}}, \\
v_{s}^{2} \vec{\nabla} \widetilde{n}_{2} \equiv \frac{1}{n m^{*}} \vec{\nabla} P ; \quad \tilde{n} \equiv \widetilde{n}_{2} \delta(x),
\end{gathered}
$$

где $v_{0}$ - дрейфовая скорость электронов в квантовой яме, $m^{*}$ — эффективная масса, $P$ давление электронного газа, $v_{p}$ - частота релаксации импульса. В двумерном электронном газе характеристическая скорость равна $v_{s} \approx \hbar n^{1 / 2} \pi^{1 / 2} / m^{*}[8]$.

В результате совместного решения уравнений (15) и уравнения Пуассона (6) получено следующее дисперсионное уравнение для гибридной волны:

$\varepsilon(\omega)-\frac{e^{2} n_{20} k /\left(2 m^{*} \varepsilon_{0}\right)}{\left(\omega-k v_{0}\right)\left(\omega-k v_{0}+\mathrm{i} v_{\mathrm{p}}\right)-v_{s}^{2} k^{2}}=0$.

\section{3. ЧИСЛЕННОЕ МОДЕЛИРОВАНИЕ ИНКРЕМЕНТОВ НЕУСТОЙЧИВОСТИ}

Неустойчивость оптических фононов имеет место, когда дрейфовая скорость электронов превосходит критическую величину $v_{0}>$ $(2 \ldots 3) v_{s} \approx(3 \ldots 5) \times 10^{7} \mathrm{~cm} / \mathrm{c}$. Этот порог обусловлен наличием электронного давления $v_{s} \neq 0$.

Неустойчивость исследована для структур с квантовыми ямами на основе $n$-InSb, InAs, GaAs. Параметры полупроводников взяты из базы данных [3]. Для практической реализации неустойчивости предпочтительны полупроводниковые структуры с селективным легиро-

ISSN 0021-3470. Радиоэлектроника. 2014. № 2. 
ванием, где достижимы чрезвычайно высокие значения подвижностей электронов, т.е. низкие значения частот столкновения $v_{\mathrm{p}} \sim 10^{10} \mathrm{c}^{-1}$.

Результаты моделирования представлены на рис. 1-3.

Неустойчивость имеет место для структур на основе всех указанных полупроводников. На рис. 1-3 приведены значения действительной (рис. $1 a-3 a)$ и мнимой (рис. 1б-3б) частей $\omega^{\prime}, \omega^{\prime \prime}$, полученных при кинетическом рассмотрении.

При моделировании приняты следующие параметры (рис. 1): полупроводник InSb, двумерная электронная концентрация $n_{02}=5 \times 10^{10}$ $\mathrm{cm}^{-2}$, дрейфовая скорость $v_{0}=10^{8} \mathrm{~cm} / \mathrm{c}$, частота столкновений $v_{\mathrm{p}}=10^{10} \mathrm{c}^{-1}, m^{*}=10^{-32} \mathrm{\kappa г,} \omega_{\mathrm{L}}=$ $3,59 \times 10^{13} \mathrm{c}^{-1}$. Рис. 2 соответствует параметрам: полупроводник InAs, $n_{02}=10^{11} \mathrm{~cm}^{-2}, v_{0}=8 \times 10^{7}$ $\mathrm{cm} / \mathrm{c}, v_{\mathrm{p}}=10^{10} \mathrm{c}^{-1}, m^{*}=2 \times 10^{-32} \mathrm{\kappa}, \omega_{\mathrm{L}}=$ $4,40 \times 10^{13} \mathrm{c}^{-1}$. Рис. 3 соответствует параметрам: полупроводник GaAs, $n_{02}=3 \times 10^{11} \mathrm{~cm}^{-2}, v_{0}=$ $6 \times 10^{7} \mathrm{~cm} / \mathrm{c}, v_{\mathrm{p}}=10^{10} \mathrm{c}^{-1}, m^{*}=6,5 \times 10^{-32} \mathrm{\kappa г,}, \omega_{\mathrm{L}}=$ $5,60 \times 10^{13} \mathrm{c}^{-1}$.

Для последнего случая, инкремент неустойчивости слабо зависит от частоты столкновения если $v_{\mathrm{p}} \leq 10^{11} \mathrm{c}^{-1}$. Для InSb и InAs для наблюдения неустойчивости возможно использовать меньшие значения двумерной электронной концентрации, но инкременты оказываются низкими и неустойчивость реализуется при высоких дрейфовых скоростях. Отметим, что в области неустойчивости выполняется соотношение $k \approx \omega^{\prime} / v_{0}$. Для усиления волны на 20-30 дБ требуются структуры длиной около $10 \mu \mathrm{M.}$

По принятой терминологии, в данной системе имеет место конвективная неустойчивость [5], поскольку отсутствуют волны, распространяющиеся в отрицательном направлении оси $O Z$. Поэтому для генерации волн следует использовать дополнительную обратную связь. Данную неустойчивость возможно использовать для пространственного усиления волн терагерцового диапазона с пространственным инкрементом усиления $\omega^{\prime \prime} / v_{0}$.

Как гидродинамический подход, так и кинетический, предсказывают неустойчивость оптических фононов, связанных с волнами пространственного заряда, т.е. в интервале значений волнового числа $k \sim(0,3-2) \times 10^{8} \mathrm{M}^{-1}$ появляются положительные значения мнимой части $\omega^{\prime \prime} \sim 10^{11}-10^{12} \mathrm{c}^{-1}$. Действительная часть $\omega^{\prime}$ соответствует частоте продольных оптических фононов $\omega_{\mathrm{L}}$ в полупроводнике $[3,15]$.

Гидродинамический метод дает значения инкрементов неустойчивости завышенные примерно в 3-5 раз. Это объясняется тем, что в кинетике проявляются дополнительные механизмы затухания волн пространственного заряда $[10,12]$. Поэтому более простой гидродинамический подход может быть использован только для качественного анализа неустойчивости.

Повышение температуры электронного газа приводит к росту тепловой скорости электронов и к росту частоты столкновений. Более того, нагрев электронного газа является механизмом стабилизации неустойчивости при ее развитии. Поэтому для избежания нагрева электронного газа генерацию оптических фононов следует реализовывать при воздействии электрического поля смещения $E_{0}$ в импульсном режиме субнаносекундной длительности $<10^{-9}$ с.

Кроме того, из-за роста частоты столкновения при более высоких уровнях легирования оптимальные значения концентрации электронов равны примерно $n_{02}=(0,3-5) \times 10^{11} \mathrm{~cm}^{-2}$ для различных полупроводниковых структур. При повышенных концентрациях $n_{02} \geq 10^{12}$ $\mathrm{cm}^{-2}$ инкременты неустойчивости увеличиваются незначительно, но тогда потребуются нереалистичные значения дрейфовых скоростей $v_{0} \geq 10^{8} \mathrm{~cm} / \mathrm{c}$.

Также рассмотрена неустойчивость при дрейфе объемного электронного газа. Теоретически, она также имеет место. Однако поскольку частоты столкновений электронов в объеме 

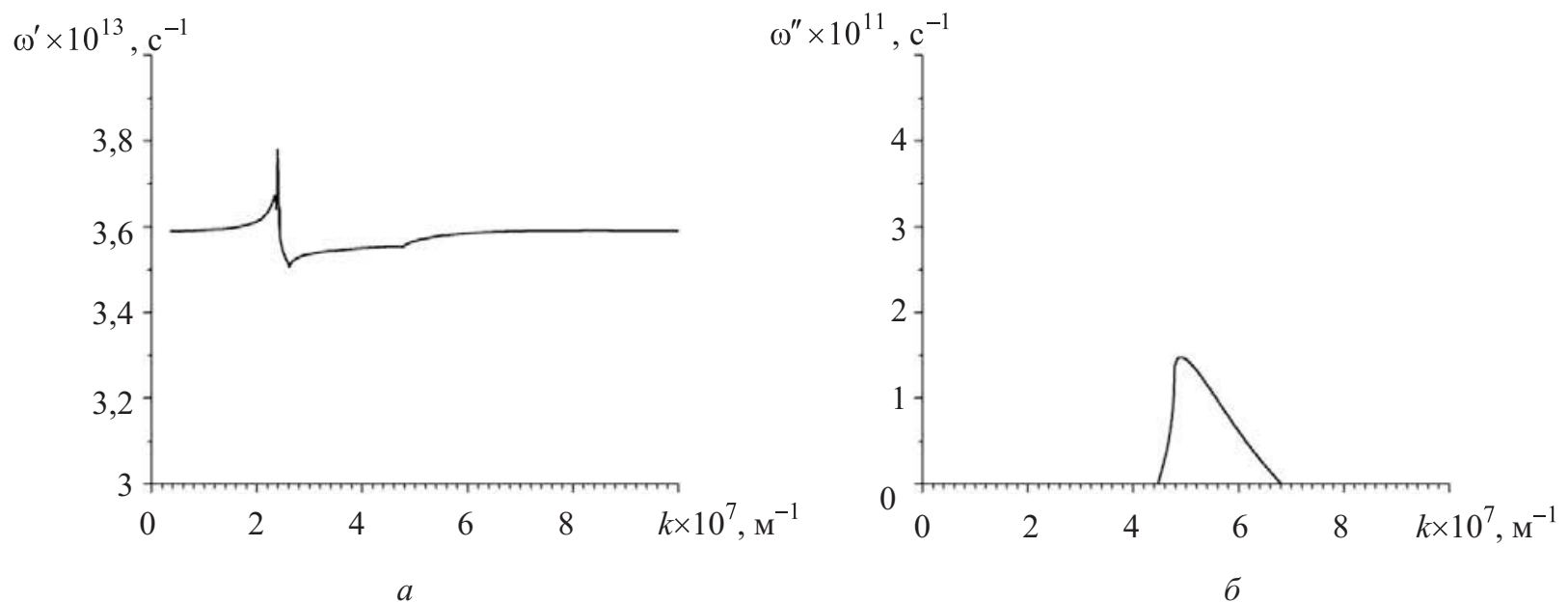

Рис. 1
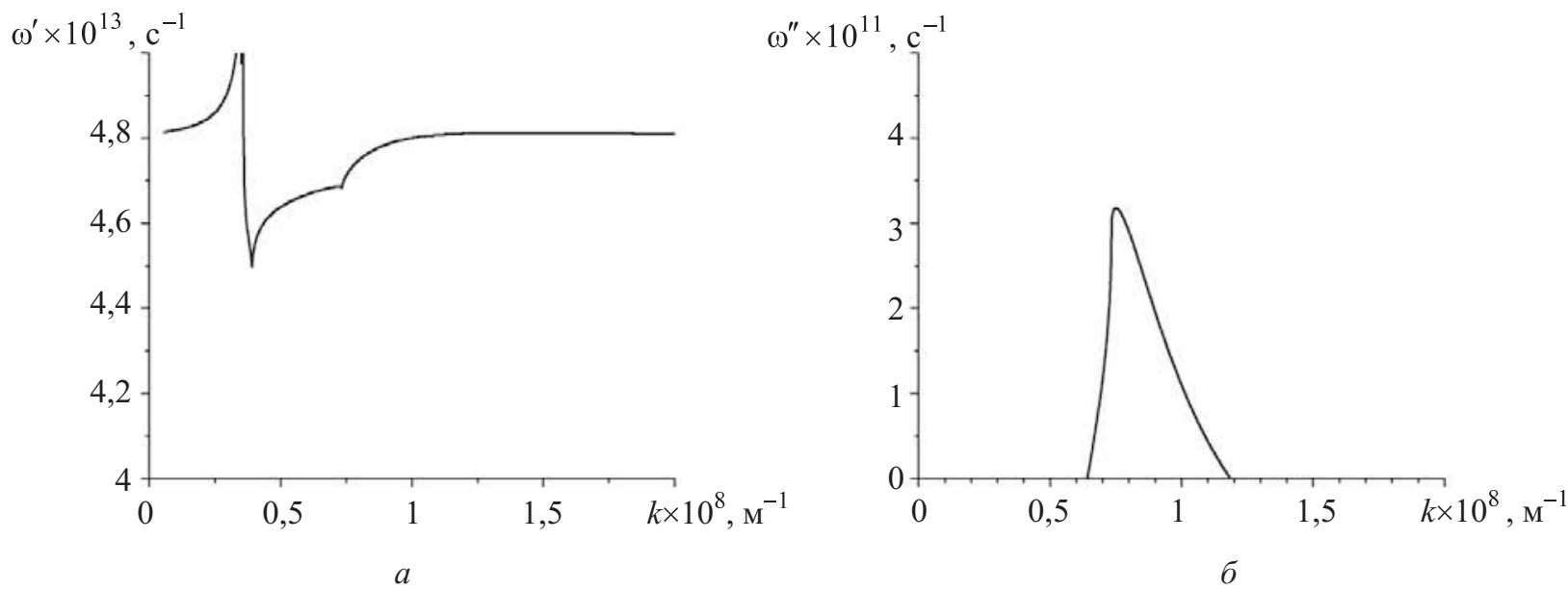

Рис. 2
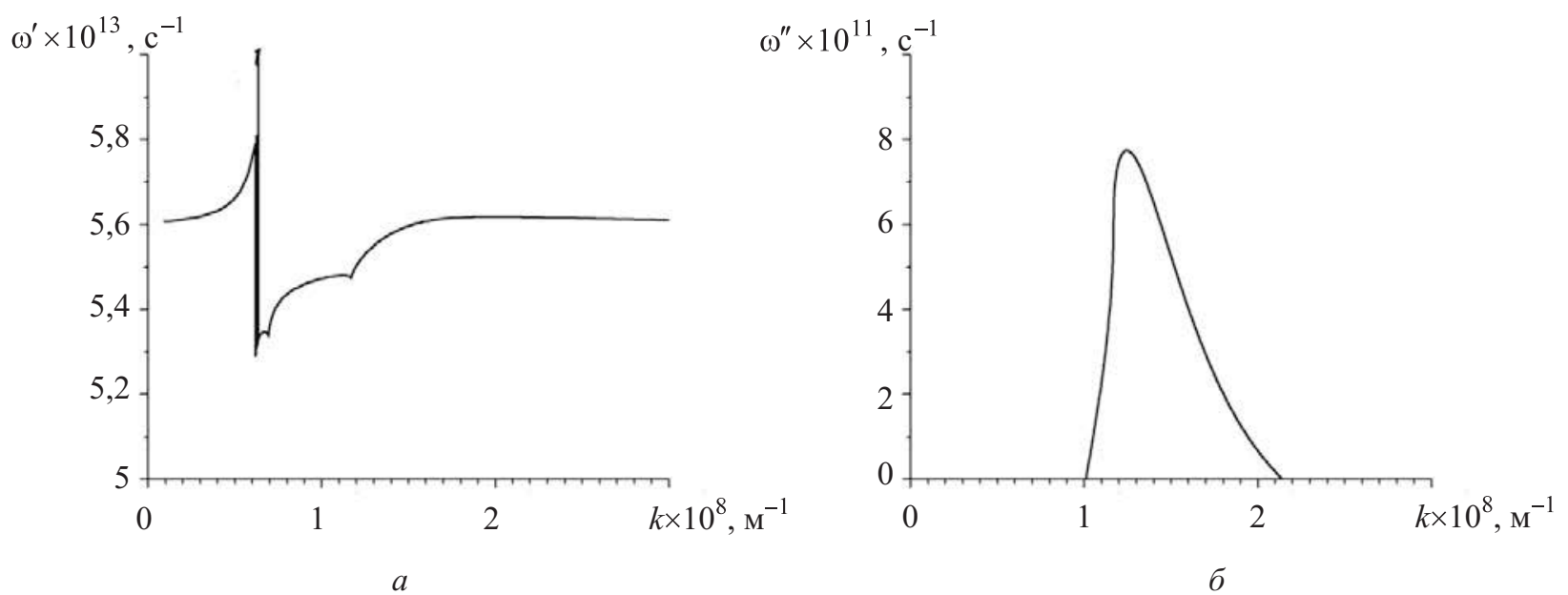

Рис. 3 
легированного полупроводника не меньше $v_{\mathrm{p}}$ $\geq 10^{11} \mathrm{c}^{-1}$, то такая неустойчивость практически нереализуема из-за сильного нагрева электронного газа.

Кроме того, структуры с квантовыми ямами имеют преимущество, связанное с тем, что двумерный электронный газ является вырожденным при более высоких температурах по сравнению с объемными полупроводниками [16]. Следовательно, неустойчивость может быть реализована при более высоких температурах.

Конструктивная часть возможного генератора рассмотрена в $[6,7]$.

\section{4. ВЫВОДЫ}

В данной работе двумя методами получены условия для реализации неустойчивостей, приводящих к усилению или генерации в терагерцовом диапазоне оптических фононов в полупроводниковых структурах с квантовыми ямами на основе полупроводников $n$-InSb, InAs, GaAs.

Механизм усиления основан на эффекте Черенкова при дрейфе двумерного электронного газа, когда скорость дрейфа электронов превосходит фазовую скорость гибридной волны в полупроводнике.

Результаты компьютерного моделирования для реальных параметров полупроводниковых структур показывают возможность наблюдения данной неустойчивости.

Квантовый кинетический подход является более адекватным, в то время как простой гидродинамический подход дает завышенные значения инкрементов неустойчивости в 3-5 раз и является только качественным.

Таким образом, неустойчивость оптических фононов существует и может быть использована на современном уровне охлаждения малогабаритными и простыми конструкциями $[6,7]$.

\section{БИБЛИОГРАФИЧЕСКИЙ СПИСОК}

1. Siegel P. H. Terahertz technology / P. H. Siegel // IEEE Trans. Microwave Theory Tech. - 2002. - Vol. 50, No. 3. - P. 910-928. - DOI : 10.1109/22.989974.

2. Kohler R. Terahertz semiconductor-heterostructure laser / Rudeger Kohler, Alessandro Tredicucci, Fabio Beltram, Harvey E. Beere, Edmund H. Linfield, A. Giles Davies, David A. Ritchie, Rita C. Iotti, Fausto Rossi // Nature. - 2002. - Vol. 417. - P. 156-162. - DOI : 10.1038/417156a.

3. Yu P. Y. Fundamentals of Semiconductors. Physics and Materials Properties / P. Y. Yu, M. Cardona. New York : Springer, 2010. — Режим доступа : http://www.ioffe.ru/SVA/NSM/.

4. Liang $W$. Observation of optical phonon instability induced by drifting electrons in semiconductor nanostructures / W. Liang, K. T. Tsen, Otto F. Sankey, S. M. Komirenko, K. W. Kim, V. A. Kochelap, Meng-Chyi Wu, Chong-Long Ho, Wen-Jeng Ho, H. Morkoc // Appl. Phys. Lett. - 2003. - Vol. 82, No. 12. — P. 1968-1970. - DOI : 10.1063/1.1563730.

5. Федорченко А. М. Абсолютная и конвективная неустойчивость в плазме и твердых телах / А. М. Федорченко, Н. Я. Коцаренко. - М. : Наука, 1981.

6. Koshevaya $S$. V. Amplification of hypersonic by GaAs crystals / S. V. Koshevaya, V. V. Grimalsky, A. Garcia-B., M. F. Diaz-A. // Ukr. J. Phys. - 2006. - Vol. 51, No. 6. - Р. 593-597. - Режим доступа : http://ujp.bitp.kiev.ua/files/journals/51/6/510610p.pdf.

7. Koshevaya $S$. $V$. Physical foundations of integrated circuits of a MM range design / S. V. Koshevaya, B. N. Emelyanenkov, L. G. Gassanov, M. Yu. Omelyanenko // Izv. Vyssh. Uchebn. Zaved., Radioelektron - 1982. - Vol. 25, No. 10. - P. 5-14.

8. Datta S. Electronic Transport in Mesoscopic Systems / S. Datta. — Cambidge : CUP, 1999.

9. Квасников И. А. Статистическая физика: Т. 2 / И. А. Квасников. - М. : Едиториал УРСС, 2002.

10. Ferry D. K. Transport in Nanostructures / D. K. Ferry, S. M. Goodnick, J. Bird. — Cambridge : CUP, 2009.

11. Александров А. Ф. Принципы электродинамики плазмы / А. Ф. Александров, Л. С. Богданкевич, А. А. Рухадзе. - М. : Высшая школа, 1990.

12. Кондратьев А. С. Лекции по теории квантовых жидкостей / А. С. Кондратьев, А. Е. Кучма. - Л. : Изд-во ЛГУ, 1989.

13. Лифшии E. М. Физическая кинетика / Е. М. Лифшиц, Л. П. Питаевский. - М. : Наука, 1979.

14. Пожела Ю. Физика быстродействующих транзисторов / Ю. Пожела. - Вильнюс : Мокслас, 1989.

15. Kossevich $A$. The crystal lattice. Phonons, solitons, dislocations / A. Kossevich. - Berlin : Wiley-VCH, 1999.

16. Шик А. Я. Физика низкоразмерных систем / А. Я. Шик, Л. Г. Бакуева, С. Ф. Мусихин, С. А. Рыков. - СПб. : Наука, 2001. 


\section{N F O R M A T ION O N T H E A R T I C L E}

\section{AMPLIFICATION OF OPTICAL PHONONS IN NARROW BAND SEMICONDUCTORS AT LOW TEMPERATURES}

Christian Castrejon-M., ccastrejon@uaem.mx, Universidad Autonoma del Estado de Morelos, Cuernavaca, Morelos, Mexico

Vladimir V. Grimalsky, v_grim@yahoo.com, Universidad Autonoma del Estado de Morelos, Cuernavaca, Morelos, Mexico

Svetlana V. Koshevaya, svetlana@uaem.mx, Universidad Autonoma del Estado de Morelos, Cuernavaca, Morelos, Mexico

Margarita Tecpoyotl-T., tecpoyotl@uaem.mx, Universidad Autonoma del Estado de Morelos, Cuernavaca, Morelos, Mexico

Terahertz radiation generation by using the natural modes of solid body has been investigated. The numerical simulation of instability of terahertz range optical phonons in semiconductor structures with quantum wells during the drift of two-dimensional electron gas was performed. The main obstacle of implementing the specified instability is the heating of electron gas during its drift. That is why the investigations were performed for low temperatures $\mathrm{T}<77 \mathrm{~K}$, and the electron gas was assumed to be degenerate. Due to the existing closed cycle microcoolers and also the feedback implementation, it is possible to experimentally observe the generation of optical phonons. The numerical simulation involved the use of both kinematic and hydrodynamic approaches. It has been shown that the kinetic approach is more adequate, while the hydrodynamic one leads to overestimated values of instability increments

Keywords: optical phonon; semiconductor structure; kinematic approach; electron gas; quantum well

\section{REFERENCES}

1. SIEGEL, P.H. Terahertz technology. IEEE Trans. Microwave Theory Tech., v.50, n.3, p.910-928, 2002. doi: http://dx.doi.org/10.1109/22.989974.

2. KOHLER, RUDEGER; TREDICUCCI, ALESSANDRO; BELTRAM, FABIO; BEERE, HARVEY E.; LINFIELD, EDMUND H.; DAVIES, A. GILES; RITCHIE, DAVID A.; IOTTI, RITA C.; ROSSI, FAUSTO. Terahertz semiconductor-heterostructure laser. Nature, v.417, p.156-162, 2002. doi: http://dx.doi.org/10.1038/417156a.

3. YU, P.Y.; CARDONA, M. Fundamentals of Semiconductors. Physics and Materials Properties. New York: Springer, 2010.

4. LIANG, W.; TSEN, K.T.; SANKEY, OTTO F.; KOMIRENKO, S.M.; KIM, K.W.; KOCHELAP, V.A.; WU, MENG-CHYI; HO, CHONG-LONG; HO, WEN-JENG; MORKOC, H. Observation of optical phonon instability induced by drifting electrons in semiconductor nanostructures, Appl. Phys. Lett., v.82, n.12, p.1968-1970, 2003. doi: http://dx.doi.org/ $10.1063 / 1.1563730$.

5. FEDORCHENKO, A.M. AND KOTSARENKO, N.Y. Absolute and Convective Instability in Plasma and Solid Bodies. Moscow: Nauka, 1981 [in Russian].

6. KOSHEVAYA, S.V.; GRIMALSKY, V.V.; GARCIA-B., A.; DIAZ-A., M.F. Amplification of hypersonic by GaAs crystals. Ukr. J. Phys., v.51, n.6, p.593-597, 2006, http://uip.bitp.kiev.ua/files/journals/51/6/510610p.pdf.
7. KOSHEVAYA, S.V.; EMELYANENKOV, B.N.; GASSANOV, L.G.; OMELYANENKO, M.Y. Physical foundations of integrated circuits of a MM range design. Izv. Vyssh. Uchebn. Zaved., Radioelektron., v.25, n.10, p.5-14, 1982 [in Russian]. Radioelectron. Commun. Syst., v.25, n.10, p.1-10, 1982.

8. DATTA, S. Electronic Transport in Mesoscopic Systems. Cambidge: CUP, 1999.

9. KVASNIKOV, I.A. Statistical Physics, v.2. Moscow: Editorial URSS, 2002 [in Russian].

10. FERRY, D.K.; GOODNICK, S.M.; BIRD, J. Transport in Nanostructures. Cambridge: CUP, 2009.

11. ALEKSANDROV, A.F.; BOGDANKEVICH, L.S.; RUKHADZE, A.A. Principles of Plasma Electrodynamics. Moscow: Vyssh. Shkola, 1990 [in Russian].

12. KONDRAT'EV, A.S. AND KUCHMA, A.E. Lectures on the Theory of Quantum Liquids. Leningrad: Izdat. LGU, 1989 [in Russian].

13. LIFSHITS, E.M. AND PITAEVSKII, L.P. Physical Kinetics. Moscow: Nauka, 1979 [in Russian].

14. POZHELA, Y. Physics of Fast Transistors. Vilnius: Mokslas, 1989 [in Russian].

15. KOSSEVICH, A. The Crystal Lattice. Phonons, Solitons, Dislocations. Berlin: Wiley-VCH, 1999.

16. SHIK, A.Y.; BAKUEVA, L.G.; MUSIKHIN, S.F.; RYKOV, S.A. Physics of Low-Dimensional Systems. St. Petersburg: Nauka, 2001 [in Russian].

Received in final form December 31, 2013 